\title{
Interacciones empaque-alimento: migración
}

\author{
Diana Paola Navia P.* \\ Alfredo Adolfo Ayala A.* \\ HéctorSamuel Villada C.**
}

Recibido: 16/12/2013 - Aceptado: 27/06/2014

\begin{abstract}
Resumen
La calidad e inocuidad de los productos alimenticios es un tema relevante que involucra el comportamiento de los empaques en los procesos de preparación y almacenamiento de alimentos. En este contexto, el cumplimiento de la legislación alimentaria, cada día más exigente, requiere el conocimiento de varios aspectos clave en los sistemas empaque-alimento. Este artículo presenta una revisión acerca de los principales materiales de empaque actualmente usados en la industria alimentaria, sus interacciones con el producto empacado principalmente la migración de macro y microelementos desde el empaque hacia el alimento, abordando técnicas analíticas y modelos matemáticos usados en la identificación de elementos migrantes, así como también aspectos relacionados con la normativa establecida nacional e internacionalmente.
\end{abstract}

Palabras clave: alimentos, empaques, interacción, migración.

\footnotetext{
Doctora en Ingeniería de Alimentos, Docente Universidad de San Buenaventura Cali, Facultad de Ingeniería, Avenida 10 de Mayo, La Umbría, Vía a Pance, Tel: 3182223, dpnavia@usbcali.edu.co

** Doctor en Ciencia y Tecnología de Alimentos, Docente Universidad del Valle, Escuela de Ingeniería de Alimentos, Calle 13 No 100-00, Tel: 3212223, alfredo.ayala@correounivalle.edu.co

*** Doctor en Ingeniería de Alimentos, Docente Universidad del Cauca, Facultad de Ciencias Agropecuarias, Departamento de Agroindustria, Vereda las Guacas, Tel: 8245976, villada@unicauca.edu.co
} 


\title{
Food packaging-interactions: migration
}

\begin{abstract}
Foodstuffs quality and safety are important issues involving packaging behavior in food preparing and storing processes. In this context, food legislation compliance increasingly demanding, concerns the knowledge of several key aspects of food-packaging systems. This article presents a review about main packaging materials currently used in the food industry, their interactions with the packaged product, mainly migration of macro and microelements from packaging to food, analytical techniques and mathematical models used in elements migrants identification, the national and international regulations established.
\end{abstract}

Keywords: foods, packaging, interaction, migration. 


\section{INTRODUCCIÓN}

Los empaques juegan un papel muy importante en la vida cotidiana de las personas, ya que son útiles en muchos campos de la industria. En el campo alimentario, estos cumplen funciones específicas tales como contener, proteger, informar y atraer [1], todo ello en aras de satisfacer las exigencias de los clientes. Es bien conocido que la presentación de los productos ante el consumidor es clave y se convierte en una estrategia de mercadeo, donde el empaque es el protagonista esencial. En este sentido, es trascendente tener en cuenta el diseño, el tipo de material usado y las características funcionales del mismo, principalmente cuando se incorpora el término "sostenibilidad del empaque" que comprende eficiencia en términos económicos, sociales y ambientales [2].

En la actualidad, se han hecho innovaciones importantes aplicando tecnologías modernas como el envasado en atmósferas modificadas [3, 4], envasado aséptico [5], empaques que soportan condiciones extremas de temperatura (cocción de alimentos en hornos microondas) [6], empaques activos [7], materiales elaborados con nanopartículas, entre otros. Desde el punto de vista ambiental, los nuevos desarrollos comprenden materiales biodegradables específicamente de fuentes naturales, como polímeros de origen vegetal, animal o microbiano [8,9]. Existen también innovaciones en los procesos de reciclaje y reutilización de materiales plásticos usados en la industria de los empaques; sin embargo, la naturaleza compleja de los plásticos en términos de composición polimérica y la presencia de impurezas demandan pre-tratamientos de limpieza y separación, previos al reciclaje [10].

La industria de alimentos y los proveedores de los materiales usados en empaques alimentarios tienen gran responsabilidad y compromiso para proporcionar alimentos inocuos a los consumidores; debido a ello es fundamental prevenir cualquier peligro procedente del empaque hacia el alimento que pueda poner en riesgo la seguridad del mismo. Por lo anterior, es pertinente considerar el comportamiento de algunos de los componentes de los materiales de empaque como aditivos, plastificantes, colorantes, antiestáticos en las interacciones con los productos empacados durante el almacenamiento. Este artículo presenta una revisión de la migración de dichos componentes, como una importante interacción alimento-empaque, algunas investigaciones realizadas en este campo y la normativa vigente relacionada.

\section{GENERALIDADES}

\subsection{Empaques usados en la industria alimentaria}

Los principales materiales usados en la industria alimentaria para empacar y/o embalar los productos son vidrio, metal, plástico, papel y cartón. Estos materiales son regulados bajo las directrices de la FDA (Food and Drug Administration) [11]. 


\subsubsection{Plástico}

La Directiva 82/711/EEC establece y define el concepto de los plástico: "compuesto macromolecular orgánico obtenido por polimerización, policondensación, poliadición u otro procedimiento similar a partir de moléculas de peso molecular inferior o por modificación química de macromoléculas naturales" [12]. Los plásticos convencionales son polímeros elaborados con materias primas a partir de fuentes de origen fósil. Los materiales de este tipo más frecuentemente usados para empaques de alimentos son polietileno (PE) de alta densidad (HDPE) y de baja densidad (LDPE), polipropileno (PP), poliestireno (PS), cloruro de polivinilo (PVC) y polietilen-tereftalato (PET) [13]. También se usa el etilen vinil alcohol (EVOH) y las poliamidas (PA) más comúnmente conocidas como nylon [11].

\subsubsection{Papel y cartón}

Las principales aplicaciones son el papel kraft (empaque de harinas, azúcar, frutas secas y hortalizas), papel sulfito (bolsas para dulces y galletas) papel resistente a la grasa (envolturas de bocadillos, galletas, dulces y otros alimentos grasosos) y papel cristal (revestimiento para galletas, comidas rápidas, y productos horneados) [11].

\subsubsection{Materiales biodegradables}

Son aquellos que se degradan por acción de los microrganismos como bacterias, hongos y algas [14]. Ejemplos de estos materiales son el ácido poliláctico (PLA), polohidroxialcanoatos (PHAs), policaprolactona (PCL), almidón, quitina y quitosano.

\section{INTERACCIONES EMPAQUE-ALIMENTO}

Las interacciones entre el empaque y el alimento pueden clasificarse de la siguiente manera [15]:

- Migración. Es la transferencia de componentes desde el empaque hacia el alimento durante su almacenamiento o preparación. [15].

- Permeación de gases y vapor de agua. Hace referencia a los procesos de transporte de gases y vapor de agua desde el interior hacia el exterior del sistema empaque/ alimento, y viceversa [15].

- Sorción y/o permeación de vapores orgánicos. Cuando el sistema empaque/ alimento se expone a olores indeseables (almacenamiento inapropiado), el empaque puede adsorberlos. Otro caso es cuando el olor deseable propio del producto empacado se pierde por la permeación del empaque [15]. 
- Transparencia de los empaques alimentarios a la luz. La luz, principalmente en longitudes de onda corta, puede catalizar reacciones adversas, como la oxidación de los alimentos. Esto puede conducir a decoloración, pérdida de nutrientes, o desarrollo de malos olores [15].

\subsection{MIGRACIÓN}

El término «migración» generalmente describe un proceso de difusión, que puede estar fuertemente influenciado por la interacción de los componentes del alimento con el material de empaque [16].

\subsubsection{Principales elementos migrantes}

Las sustancias que pueden migrar al alimento dependen de la naturaleza del material de empaque. Los polímeros usados en empaques y embalajes alimentarios están formados por monómeros, oligómeros, aditivos y residuos de solventes (tintas y/o adhesivos) que pueden transferirse al alimento [16, 17]. Dentro de los aditivos usados en la fabricación de empaques están los plastificantes, antioxidantes, estabilizantes y colorantes, entre otros [16].

Los monómeros y oligómeros (número finito de monómeros) son sustancias reactivas y potencialmente tóxicas. Ejemplo de ellos son los monómeros estireno y cloruro de vinilo usados para elaborar PS y PVC, respectivamente [16].

Los plastificantes (estearato de butilo, acetiltributil citrato y adipatos, entre otros) presentan baja toxicidad, pero tienen un efecto potencial carcinogénico [16]. La migración de plastificantes aumenta cuando hay contacto directo con alimentos grasos y con el incremento de temperatura [16].

Los antioxidantes se utilizan para disminuir el proceso de oxidación de los plásticos generado por la exposición a la luz. El BHT (Butil hidroxitolueno) y el Irganox 1010 son los antioxidantes más utilizados. La mayoría de los antioxidantes son tóxicos [16]. De otro lado, para prevenir la foto-oxidación de los materiales poliméricos, se adicionan estabilizantes de luz como las HALS (Aminas estéricamente impedidas) [15].

Los colorantes usados pueden dividirse en dos grandes categorías: pigmentos y tintas. Los pigmentos pueden ser orgánicos e inorgánicos, y se caracterizan por tener alta incompatibilidad con los materiales poliméricos por lo que se requieren métodos de mezclado intensos [15]. Las tintas presentan alta compatibilidad con la mayoría de matrices poliméricas, se funden fácilmente y no afectan la transparencia del material [15]. 
Las sustancias que no son adicionadas de forma intencional en el proceso de producción de los empaques, como las que se originan producto de los procesos de descomposición de los mismos (solventes: residuos de tintas de impresión y adhesivos), también pueden migrar al alimento bajo condiciones específicas de almacenamiento [18].

\subsubsection{Simulantes de alimentos}

Un simulante es un producto que imita el comportamiento de un alimento o grupo de alimentos. Dada la complejidad de los productos alimenticios y la variedad de condiciones que surgen del contacto con los plásticos, se han establecido oficialmente ciertas sustancias listadas en la tabla 1, como los simulantes posibles a usar en la determinación de migración en alimentos. Cuando no es posible usar ninguno de los simulantes grasos, se permite el uso de iso-octano, etanol al $95 \%$ u óxido de poli-fenileno (Tenax) [19].

Tabla 1. Simulantes alimentarios

\begin{tabular}{|c|l|}
\hline Tipo & \multicolumn{1}{|c|}{ Simulante } \\
\hline \multirow{4}{*}{ Acuosos } & A: Agua destilada \\
\cline { 2 - 2 } & B: Ácido acético $3 \%(\mathrm{p} / \mathrm{v}) \mathrm{B}$ \\
\cline { 2 - 2 } & C: Etanol al $15 \%(\mathrm{v} / \mathrm{v})$ \\
\hline \multirow{3}{*}{ Grasos(D) } & Aceite de oliva rectificado \\
\cline { 2 - 2 } & Aceite de girasol \\
\cline { 2 - 2 } & HB307 \\
\hline
\end{tabular}

Fuente: $[26,29]$

\subsubsection{Simulantes de contaminantes}

Debido a los múltiples componentes que posee, es complejo determinar el tipo de contaminante en un material reciclado. Por esta razón, la FDA propuso un método consistente en incluir un contaminante (simulante) en la resina a reprocesar y realizar el proceso de descontaminación (depolimerización, pos-condensación, uso de barreras funcionales) para verificar la efectividad del proceso. Finalmente se realizan pruebas con simulantes de alimentos para evaluar la migración del componente problema (contaminante) [20].

\subsubsection{Modelos matemáticos}

El uso de modelos matemáticos para predecir el comportamiento de la migración puede reducir gran cantidad de pruebas y ensayos de laboratorio que suelen ser largos y dispendiosos [21]. 
Debido a que el proceso de migración es netamente difusional, la ecuación más comúnmente aplicada es la de la ley de fick (Ec. 1) [21]:

$$
\frac{\partial C_{A}^{P}}{\partial t}=D_{A}^{P} \frac{\partial^{2} C_{A}^{P}}{\partial x^{2}}
$$

Donde " $C_{A}^{P}$ " representa la concentración de la especie migrante "A" en el empaque "P", " $t$ " es el tiempo, " $x$ " la dimensión lineal de la migración y " $D_{A}^{P}$ " es la difusión de "A" en "P".

En ausencia de reacciones químicas o evaporación, la ecuación general de balance de masa (Ec. 2 y 3) es: la cantidad inicial de "A" presente en el material de empaque "P" es igual a la suma de la cantidad total que migra en el alimento después del tiempo " $t$ " más la cantidad remanente en el empaque. Esto es válido en cualquier instante y en el equilibrio [22], así.

$$
\begin{aligned}
& M_{A}^{P}(0)=M_{A}^{P}(t)+M_{A}^{F}(t) \\
& M_{A}^{P}(0)=M_{A}^{P}(\infty)+M_{A}^{F}(\infty)
\end{aligned}
$$

Donde " $M_{A}^{i}(t)$ " representa la masa de "A" en la fase " $i$ " (P empaque, F alimento) en el tiempo " $t$ ". En el equilibrio, se definen las constantes " $K p$ ” y “ $\alpha$ ” [21],

$$
\begin{aligned}
& K_{p}=\frac{C_{A}^{P}(\infty)}{C_{A}^{F}(\infty)} \\
& \alpha=\frac{M_{A}^{F}(\infty)}{M_{A}^{P}(\infty)}=\frac{C_{A}^{F}(\infty) V^{F}}{C_{A}^{P}(\infty) V^{P}}=\frac{V^{F}}{K_{P} V^{P}}
\end{aligned}
$$

Donde " $K p$ " es el coeficiente de partición de "A" en el sistema empaque/alimento y " $V$ " es el volumen de la fase " $i$ ". Para el caso en el que el proceso de migración es controlado por el mecanismo de difusión del migrante a través del material de empaque y, además, el migrante esté bien distribuido en el alimento [21], se pueden usar el modelo de Piringer (Ec. 6) y el modelo de FDA (Ec. 7) [21]:

$$
\frac{M_{A}^{F}}{M_{A}^{F}(\infty)}=1-\frac{8}{\pi^{2}} \sum_{n=0}^{\infty} \frac{1}{(2 n+1)^{2}} \exp \left(-D_{A}^{P} t \frac{(2 n+1)^{2} \pi^{2}}{L^{2}}\right)
$$




$$
\frac{M_{A}^{F}}{M_{A}^{F}(0)}=\frac{2}{L \sqrt{\pi}} \sqrt{D_{A}^{P} t}
$$

Estos modelos han sido evaluados por varios autores. Un estudio en el que fueron comparados los valores de migración obtenidos con el modelo Piringer y los experimentales reportó que dicho modelo sobre-estimó los valores experimentales sobre todo a bajas temperaturas [23]. En otro estudio se encontró que para el $95 \%$ de los casos de migración evaluados, el modelo Piringer generó valores más altos que los valores reales de migración [24].

\subsubsection{Normativa}

Con el objetivo de unificar la legislación y facilitar la protección a los consumidores la comisión de la comunidad europea (EC) y la FDA implementaron una serie de directivas [16] para el manejo de técnicas de análisis, límites permisibles de sustancias migrantes, materiales permitidos en la elaboración de empaques para alimentos, entre otras. Las directivas relacionadas con migración están divididas en tres categorías [16]:

1. Directivas aplicables a todos los materiales y artículos.

2. Directivas aplicables a una categoría de materiales y artículos.

3. Directivas relacionadas con sustancias específicas.

Los límites establecidos para determinar la migración son: migración global o total (QM, Quantity in Material) y migración específica (SML Specific Migration Limit) [16]. La Directiva 90/128/EEC establece que el valor de migración total de un empaque hacia un alimento no debe superar los $10 \mathrm{mg} / \mathrm{dm}^{2}$ (60mg/Kg de alimento) para las sustancias permitidas como migrantes, establecidas en la mencionada directiva [16]. Existen algunas resinas usadas en la elaboración de empaques como el bisfenol A diglicidiléter (BADGE),el cual se permite en $1 \mathrm{mg} / \mathrm{kg}$ de material plásticos usado en contacto con alimentos y establece un límite de migración «no detectable» evaluado en alimentos o simulantes de alimentos con un límite de detección máximo de 0.020 $\mathrm{mg} / \mathrm{kg}$ [16]. La Directiva 85/572/EEC establece la lista de simulantes de alimentos usados para las pruebas de migración en contacto con los materiales de empaque [16]. En Colombia el Instituto Colombiano de Normas Técnicas (ICONTEC) estableció la normativa relacionada con migración. La tabla 2 presenta algunas de las normas emitidas [25-28]. 
Tabla 2. Normativa colombiana

\begin{tabular}{|l|l|}
\hline Norma NTC & \multicolumn{1}{c|}{ Contenido } \\
\hline 5022 & $\begin{array}{l}\text { Materiales y artículos plásticos destinados a estar en contacto con alimentos y bebidas. } \\
\text { Determinación de la migración global }\end{array}$ \\
\hline 5023 & Materiales compuestos y artículos plásticos para uso en contacto con alimentos y bebidas \\
\hline 4447 & $\begin{array}{l}\text { Análisis sensorial. determinación del efecto del empaque sobre los alimentos y bebidas } \\
\text { durante el almacenamiento }\end{array}$ \\
\hline 4606 & Plásticos. Determinación de la migración de los plastificantes \\
\hline
\end{tabular}

Fuente: elaboración propia

\section{INVESTIGACIONES EN MIGRACIÓN DE EMPAQUE-ALIMENTO}

En un gran número de estudios se han evaluado migrantes en varios tipos de materiales de empaque aproximadamente desde los años setenta:

- En el año de 1989, Kim et al. evaluaron el proceso de vulcanización del caucho y encontraron que las materias primas sometidas a dicho proceso pueden generar sustancias como fenantreno, antraceno y pireno las cuales se convierten en migrantes potenciales cuando el producto se encuentra en contacto con alimentos u otro tipo de productos [29].

- En la década de los 90, se evaluó la migración de oligómeros presentes en el PET cristalizado (CPET) usado comúnmente en contenedores para cocción de alimentos en microondas, y se encontró que en palomitas de maíz dichos oligómeros alcanzaron niveles de migración de $\left(0.485 \mathrm{mg} / \mathrm{dm}^{2}\right)$; sin embargo, existe la posibilidad de que otros oligómeros no detectados por el HPLC estuvieran presentes en el alimento [30].

- Aurela et al, evaluaron la migración de alquilbencenos (solvente usado en la tinta de impresión) desde empaques de cartón en hamburguesas, y encontraron valores de migración global inferiores a los permitidos [31].

- La migración de iones $\mathrm{Na}$ y $\mathrm{Cl}$ presentes en eliminadores de oxígeno introducidos en empaques plásticos de alimentos fue evaluada y se encontró que los valores de migración global fueron superiores a los permitidos en los simulantes no ácidos empelados [32].

- Conservantes convencionales (benzoato de sodio, nitrito de sodio, sorbato de potasio y lactato de sodio) fueron incorporados en películas de LDPE, y láminas de PS y PET. La migración total en simulantes mostró que se excedieron los límites permisibles [33]. 
- Un estudio evaluó la migración global de películas de PVC en varios simulantes grasos. Se encontró que el etanol del $95 \%$ fue el mejor simulante alternativo de alimentos grasos para reproducir la migración global de PVC; también se encontró que cuando el sistema empaque/alimento fue sometido a calentamiento por microondas la migración global incrementó hasta $45 \mathrm{mg} / \mathrm{dm}^{2}$, superando el límite permitido [34].

- La migración de metales ( $\mathrm{Mg}, \mathrm{Fe}$ y $\mathrm{Si}$ ) fue evaluada en películas nanocompuestas de almidón y arcilla usadas para empacar lechuga y espinaca. Los alimentos presentaron migración específica baja (inferior a la permitida). El mayor contenido de silicio observado podría ser atribuido al hecho de que las nanopartículas de arcilla se componen principalmente de este elemento [35].

- Investigadores evaluaron la migración de monómeros en películas de PLA y PLA/ PCL, y encontraron que el ácido láctico migró en cantidades superiores a las permitidas para el límite global establecido [36].

- Entre los estudios recientes en migración se destacan las valoraciones de solventes, conservantes, ácido láctico (monómero del PLA), compuestos activos y contaminantes químicos. Otros componentes como los ftalatos son usualmente incorporados como plastificantes en los plásticos convencionales y su efecto es nocivo para la salud. Al respecto se reporta migración de ftalatos en empaques alimentarios y su variación con la polaridad y la volatilidad [37].

- Otros investigadores reportaron la migración de etil lauril arginato ELA en conjunto con componentes (impurezas) propios del material de empaque "PET" en filetes de pollo y simulantes alimentarios. Los ELA son surfactantes muy utilizados en la producción de empaques alimentarios activos, ya que tienen una fuerte actividad antimicrobiana contra un amplio rango de microrganismos patógenos, especialmente en la reducción de Listeria monocytogenes. Los componentes que migraron no causaron efectos adversos en la salud [38].

- Aunque el ácido láctico (monómero de PLA) es aceptado por la FDA y la EC en la lista de sustancias permitidas para elaborar empaques en contacto con alimentos, se ha demostrado que causa irritaciones en lactantes [39]. Investigadores identificaron los productos de migración en láminas de PLA, que se utilizan en empaques de almuerzos en Japón. A $60{ }^{\circ} \mathrm{C}$, por encima de la temperatura de transición vítrea del polímero, este se descompuso, y la migración global (monómero ácido láctico y oligómeros) superó los límites permitidos. Se concluyó que la tasa de migración aumenta por las altas temperaturas [39]. 
- Contaminantes químicos como los fluoro-químicos presentes en empaques de papel fueron evaluados en palomitas de maíz preparadas en microondas, las cuales presentaron valores de migración total de $3.2 \mathrm{mg} / \mathrm{kg}$ luego de su elaboración. El estudio también concluyó que para alimentos grasos evaluados, los contenidos de emulsificantes potenciaron la migración de los químicos [40]. De otro lado, una investigación en Italia reveló que el di-isobutil ftalato (DIBP) migró desde el empaque (cajas de pizza) hacia el producto contenido con un valor de QM de 2.79 $\mathrm{ug} / \mathrm{dm}^{2}$. La valoración se realizó mediante la extracción del migrante en el espacio de cabeza del empaque [41]. El DIBP no está registrado como sustancia permitida por la FDA y la EC y puede causar problemas e irrumpir en las actividades del sistema endocrino [41].

\subsection{Tendencias}

Las investigaciones relacionadas con las interacciones empaque/alimento se están orientando en aprovechar las capacidades de migración de los materiales de empaque para obtener migraciones deseables con fines específicos, con los llamados "empaques activos", en los que el agente activo puede hacer parte del material de empaque desde su proceso de elaboración o adicionarse en forma individual haciendo parte del contenido del empaque; a esto se le conoce como migración positiva [42]. Los últimos desarrollos en empaques activos incluyen disminuir la velocidad de oxidación, crecimiento microbiano, y respiración. Otras tecnologías involucran adsorbedores/emisores de dióxido de carbono, adsorbedores/emisores de olores y sabores, y eliminadores de etileno y humedad [43]. La migración de sabores indeseables desde el empaque hacia el producto o la migración de sabores volátiles desde el producto hacia el empaque es comúnmente conocida como "flavor scalping" y ocasiona altas pérdidas de calidad en los productos empacados. Por ello, el uso de adsorbedores o eliminadores de olores/ sabores o componentes indeseables del producto empacado se ha convertido en tema de interés [44]. El uso de materiales inertes para elaborar empaques con cierres herméticos también es frecuente para contener sabores volátiles de los productos; sin embargo, todos los componentes complementarios del empaque como etiquetas y adhesivos son susceptibles de generar migración hacia el producto empacado, por lo que actualmente se desarrollan estudios orientados hacia ello [45].

De otro lado la nanotecnología es una de las herramientas actualmente más investigadas, con la que se pueden obtener condiciones muy favorables con respecto a los inconvenientes de la migración. Existen muchas investigaciones en las que los materiales de empaque biodegradables o convencionales nanocompuestos han contribuido en la disminución de la tasa de migración [46-48]. 


\section{CONCLUSIONES}

La concentración y la velocidad de migración de los elementos dependen de variables como área de contacto entre el alimento y el empaque, tiempo de contacto, temperatura de almacenamiento, composición del alimento, concentración inicial del migrante en el empaque, solubilidad y polaridad del mismo, y morfología del material de empaque, entre otros.

Indudablemente la migración debe considerarse un factor de riesgo toxicológico para los alimentos. En este sentido es importante que se continúe el apoyo de los fabricantes y proveedores de materiales de empaque, porque de los insumos y materias primas de dichos materiales, parte toda la cadena migratoria de los componentes.

Las investigaciones encaminadas en materiales nanocompuestos son promisorias, más aún las enfocadas en la elaboración de materiales biodegradables, teniendo en cuenta que mejoran las propiedades funcionales de los mismos en su aplicación como empaques de alimentos, a la vez que desarrollan productos amigables ambientalmente.

\section{REFERENCIAS}

[1] L. Duizer, T. Robertson, and J. Han, "Requirements for packaging from an ageing consumer's perspective", Packaging Technology and Science, vol. 22, n. ${ }^{\circ}$ 4, pp. 187-197, 2009.

[2] N. Nordin, and S. Selke, "Social aspect of sustainable packaging", Packaging Technology and Science, vol. 23, n. ${ }^{\circ}$, pp. 317-326, 2010.

[3] Y. Li et al., "Effect of active modified atmosphere packaging with different initial gas compositions on nutritional compounds of shiitake mushrooms (Lentinus edodes)", Postharvest Biology and Technology, vol. 92, n. ${ }^{\circ}$ 1, pp. 107-113, 2014.

[4] M. Mastromatteo et al., "Combined effect of active coating and modified atmosphere packaging on prolonging the shelf life of low-moisture Mozzarella cheese", Journal of Dairy Science, vol. 97, n. $^{\circ} 1$, pp. 36-45, 2014.

[5] C. Morris, A. Brody and, L. Wicker, "Non-thermal food processing/preservation technologies: A review with packaging implications", Packaging Technology and Science, vol. 20, n. ${ }^{\circ} 4$, pp. 275-286, 2007.

[6] M. Jamshidian et al., "Poly-Lactic Acid: production, applications, nanocomposites, and release studies", Comprehensive Reviews in Food Science and Food Safety, vol. 9, n. ${ }^{\circ}$ 5, pp. 552-571, 2010.

[7] M. Galdi and L. Incarnato, "Influence of composition on structure and barrier properties of active PET films for food packaging applications", Packaging Technology and Science, vol. 24, n. 2 , pp. 89-102, 2011. 
[8] K. Matsumoto and S. Taguchi, "Enzyme and metabolic engineering for the production of novel biopolymers: crossover of biological and chemical processes", Current Opinion in Biotechnology, vol. 24, n. ${ }^{\circ}$, pp. 1054- 1060, 2013.

[9] M. Yates and C. Barlow, "Life cycle assessments of biodegradable, commercial biopolymersA critical review”, Resources, Conservation and Recycling, vol. 78, n. ${ }^{\circ}$ 1, pp. 54-66, 2013.

[10] D. Briassoulis, M. Hiskakis and E. Babou, "Technical specifications for mechanical recycling of agricultural plastic waste”, Waste Management, vol. 33, n. ${ }^{\circ}$ 6, pp. 1516-1530, 2013.

[11] K. Marsh, and, B. Bugusu, "Food Packaging—Roles, materials and environmental issues", Journal of Food Science, vol. 72, n. ${ }^{\circ}$ 3, pp. R39-R55, 2007.

[12] I. Arvanitoyannis, S. Choreftaki and P. Tserkezou, "An update of EU legislation (Directives and Regulations) on food-related issues (Safety, Hygiene, Packaging, Technology, GMOs, Additives, Radiation, Labelling): presentation and comments", International Journal of Food Science and Technology, vol. 40, n. ${ }^{\circ}$ 10, pp. 1021-1112, 2005.

[13] N. Peelman et al., "Application of bioplastics for food packaging”, Trends in Food Science \& Technology, vol. 32, n. ${ }^{\circ}$ 2, pp. 128-141, 2013.

[14] J. Rhim, H. Park and C. Ha, "Bio-nanocomposites for food packaging applications", Progress in Polymer Science, vol. 38, n. ${ }^{\circ}$ 10-11, pp. 1629-1652, 2013.

[15] J. Hotchkiss, "An overview of food and food packaging interactions", en Food and Packaging Interactions ACS Symposium Series, vol. 365; J. Hotchkiss, ed., pp. 1-10, Washington, DC: American Chemical Society, 1988.

[16] I. Arvanitoyannis and L. Bosnea, "Migration of substances from food packaging materials to foods", Critical Reviews in Food Science and Nutrition, vol. 44, n. ${ }^{\circ}$ 2, pp. 63-76, 2004.

[17] J. Alin, and M. Hakkarainen, "Migration from polycarbonate packaging to food simulants during microwave heating", Polymer Degradation and Stability, vol. 97, n. ${ }^{\circ}$, pp. 1387-1395, 2012 .

[18] H. Ayala, O. Núñez and P. Lucci, "Recent advances in LC-MS analysis of food-packaging contaminants", Trends in Analytical Chemistry, vol. 42, n. ${ }^{\circ}$ 1, pp. 99-124, 2013.

[19] L. Reinas et al., "Migration of two antioxidants from packaging into a solid food and into Tenax ${ }^{\circledR}$ ", Food Control, vol. 28, n. ${ }^{\circ}$ 2, pp. 333-337, 2012.

[20] E. Fasano et al., "Migration of phthalates, alkylphenols, bisphenol A and di(2-ethylhexyl) adipate from food packaging", Food Control, vol. 27, n. ${ }^{\circ}$ 1, pp. 132-138, 2012.

[21] M. Poças, "A critical survey of predictive mathematical models for migration from packaging", Critical Reviews in Food Science and Nutrition, vol. 48, n. ${ }^{\circ}$ 10, pp. 913-928, 2008.

[22] M. Canosa, Desarrollo de metodología analítica para determinación de Triclosán y Parabenes. Aplicación al estudio de su distribución y transformación en muestras ambientales, Tesis doctoral, Universidad Santiago de Compostela, España, 2009, 287 p. 
[23] Z., Wang, P. Wang, and C. Hu, "Molecular dynamics simulation on diffusion of 13 kinds of small molecules in polyethylene terephthalate", Packaging Technology and Science, vol. 23, n. 8 , pp. 457-469, 2010.

[24] T. Begley et al., "Evaluation of migration models that might be used in support of regulations for food-contact plastics" Food Additives and Contaminants, vol. 22, n. ${ }^{\circ}$ 1, pp. 73-90, 2005.

[25] Instituto Colombiano de Normas Técnicas y Certificación, ICONTEC. NTC 4447. Análisis sensorial. Determinación del efecto del empaque sobre los alimentos y bebidas durante el almacenamiento. Colombia: ICONTEC, 2007, 8 p.

[26] Instituto Colombiano de Normas Técnicas y Certificación, ICONTEC. NTC 4606. Plásticos. Determinación de la migración de los plastificantes. Colombia: ICONTEC, 1999, 5 p.

[27] Instituto Colombiano de Normas Técnicas y Certificación, ICONTEC. NTC 5022. Materiales y artículos plásticos destinados a estar en contacto con alimentos y bebidas. Determinación de la migración global Colombia: ICONTEC, 2010, 15 p.

[28] Instituto Colombiano de Normas Técnicas y Certificación, ICONTEC. NTC 5023. Materiales compuestos y artículos plásticos para uso en contacto con alimentos y bebidas. Colombia: ICONTEC, 2002, $16 \mathrm{p}$.

[29] H. Kim, S. Gilbert, and J. Johnson, "Determination of potential migrants from rubber gaskets of commercial aerosol valves used in food packaging", Journal of Food Science, vol. 54, n. ${ }^{\circ}$ 2, pp. 465-467, 1989.

[30] T. Begley, J. Dennison and H. Hollifield, "Migration into food of polyethylene terephthalate (PET) cyclic oligomers from PET microwave susceptor packaging" Food Additives and Contaminants, vol. 7, n. ${ }^{\circ}$, pp. 797-803, 1990.

[31] B, Aurela, T. Ohra-Aho and L. Soderhjelm, "Migration of alkylbenzenes from packaging into food and tenax", Packaging Technology and Science, vol. 14, no. 2, pp. 71-77, 2001.

[32] J. López-Cervantes et al., "Evaluating the migration of ingredients from active packaging and development of dedicated methods: a study of two iron-based oxygen absorbers", Food Additives and Contaminants, vol. 20, n. ${ }^{\circ}$ 3, pp. 291-299, 2003.

[33] J. Vartiainen, et al., "Properties of antimicrobial plastics containing traditional food preservatives”, Packaging Technology and Science, vol. 16, n. ${ }^{\circ}$ 6, pp. 223-229, 2003.

[34] M. Galotto and A. Guarda, "Suitability of alternative fatty food simulants to study the effect of thermal and microwave heating on overall migration of plastic packaging", Packaging Technology and Science, vol. 17, n. ${ }^{\circ}$ 4, pp. 219-223, 2004.

[35] M. Avella et al., "Biodegradable starch/clay nanocomposite films for food packaging applications”, Food Chemistry, vol. 93, n. 3 , pp. 467-474, 2005.

[36] D. Plackett et al., "Characterization of L-polylactide and L-polylactide-polycaprolactone copolymer films for use in cheese packaging applications", Packaging Technology and Science, vol. 19, n. ${ }^{\circ}$ 1, pp. 1-24, 2006. 
[37] M. Pocas et al., "Modelling migration from paper into a food simulant", Food Control, vol. 22 , n. ${ }^{\circ}$, pp. 303-312, 2011.

[38] M. Aznar et al., "Migrants determination and bioaccessibility study of ethyl lauroyl arginate (LAE) from a LAE based antimicrobial food packaging material", Food and Chemical Toxicology, vol. 56, n. ${ }^{\circ}$, pp. 363-370, 2013.

[39] M. Mutsuga, Y. Kawamura and K. Tanamoto, "Migration of lactic acid, lactide and oligomers from polylactide food-contact materials", Food Additives and Contaminants, vol. 25, n. ${ }^{\circ} 10$, pp. 1283-1290, 2008.

[40] T. Begley et al., "Migration of fluorochemical paper additives from food-contact paper into foods and food stimulants", Food Additives and Contaminants, vol. 25, n. ${ }^{\circ}$, pp. 384-390, 2008 .

[41] M. Bononi and F. Tateo, "Identification of diisobutyl phthalate (DIBP) suspected as possible contaminant in recycled cellulose for take-away pizza boxes", Packaging Technology and Science, vol. 22, n. ${ }^{\circ}$, pp. 53-58, 2009.

[42] D. Pezo et al., "Analytical procedure for the determination of Ethyl Lauroyl Arginate (LAE) to assess the kinetics and specific migration from a new antimicrobial active food packaging", Analytica Chimica Acta, vol. 745, n. ${ }^{\circ}$ 1, pp. 92-98, 2012.

[43] R. Singh, S. Giri and N. Kotwaliwale, "Shelf-life enhancement of green bell pepper (Capsicum annuum L.) under active modified atmosphere storage", Food Packaging and Shelf Life, vol. 1, n. ${ }^{\circ}$, pp. 101-112, 2014.

[44] C. López et al., "Food applications of active packaging EVOH films containing cyclodextrins for the preferential scavenging of undesirable compounds", Journal of Food Engineering, vol. 104 , n. $^{\circ} 3$, pp. 380-386, 2011

[45] P. Vera, E. Canellas and C. Nerín, "Migration of odorous compounds from adhesives used in market samples of food packaging materials by chromatography olfactometry and mass spectrometry (GC-O-MS)", Food Chemistry, vol. 145, n. 1, pp. 237-244, 2014.

[46] E. Fortunati et al., "Combined effects of cellulose nanocrystals and silver nanoparticles on the barrier and migration properties of PLA nano-biocomposites", Journal of Food Engineering, vol. 118, n. ${ }^{\circ}$, pp. 117-124, 2013.

[47] T. Duncan, "Applications of nanotechnology in food packaging and food safety: Barrier materials, antimicrobials and sensors", Journal of Colloid and Interface Science, vol. 363, n. ${ }^{\circ}$, pp. 1-24, 2011.

[48] M. Busolo and J. Lagaron, "Oxygen scavenging polyolefin nanocomposite films containing an iron modified kaolinite of interest in active food packaging applications", Innovative Food Science and Emerging Technologies, vol. 16, n. ${ }^{\circ}$ 1, pp. 211-217, 2012. 